\title{
Enhanced backward wave propagation in evanescent waveguides loaded with split ring resonators
}

Cite as: J. Appl. Phys. 102, 044902 (2007); https://doi.org/10.1063/1.2769141

Submitted: 18 January 2007 . Accepted: 28 June 2007 . Published Online: 20 August 2007

J. Carbonell, L. J. Roglá, V. E. Boria, and R. Marqués

\section{ARTICLES YOU MAY BE INTERESTED IN}

Electric-field-coupled resonators for negative permittivity metamaterials Applied Physics Letters 88, 041109 (2006); https://doi.org/10.1063/1.2166681

Split ring resonator-based left-handed coplanar waveguide

Applied Physics Letters 83, 4652 (2003); https://doi.org/10.1063/1.1631392

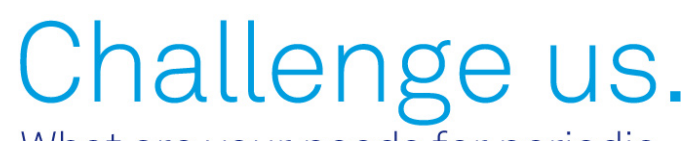

What are your needs for periodic

signal detection?

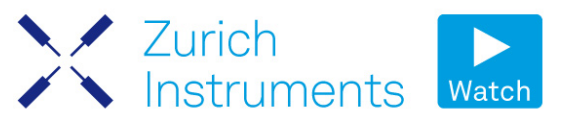

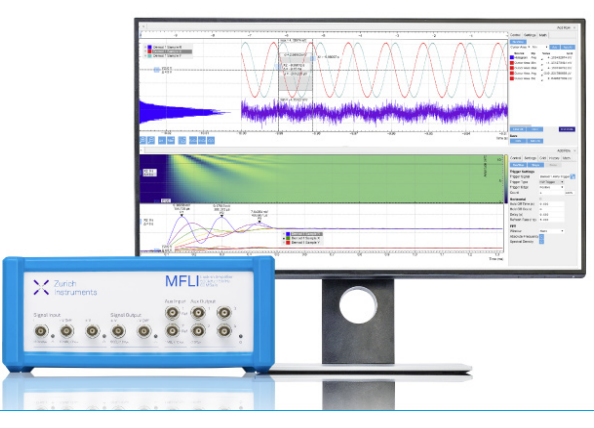

102,044902 


\title{
Enhanced backward wave propagation in evanescent waveguides loaded with split ring resonators
}

\author{
J. Carbonell, ${ }^{\text {a) }}$ L. J. Roglá, and V. E. Boria ${ }^{\text {b) }}$ \\ Instituto de Telecomunicaciones y Aplicaciones Multimedia (iTEAM), Universidad Politécnica \\ de Valencia, Camino de Vera s/n, 46022 Valencia, Spain \\ R. Marqués ${ }^{c)}$ \\ Departamento de Electrónica y Electromagnetismo, Universidad de Sevilla, Av. Reina Mercedes s/n, 41012 \\ Sevilla, Spain
}

(Received 18 January 2007; accepted 28 June 2007; published online 20 August 2007)

\begin{abstract}
This paper reports on the design and experimental characterization of evanescent waveguides loaded with split ring resonators (SRRs) supporting enhanced backward wave propagation. With respect to previous negative refractive index devices based on metallic waveguides, the use of a direct coaxial probe excitation inside an evanescent waveguide drastically improves transmission and reflection characteristics of the periodic structure. Moreover, the addition of metallic windows interleaved between consecutive resonators offers an extra degree of freedom in order to control interresonator couplings, and hence, device parameters like overall transmission, reflection, and bandwidth of the backward wave pass band. A numerical and experimental analysis of different structures overcoming the high loss issue of previously published results is detailed. An experimental validation of the proposed enhancements is done for periodic structures, where no individual tuning of the resonator sections is performed. The proposed technology mainly offers the advantage of miniaturization, and it could be combined in a further step with standard design techniques to provide frequency selective devices. (C) 2007 American Institute of Physics.
\end{abstract}

[DOI: $10.1063 / 1.2769141]$

\section{INTRODUCTION}

In the last few years, negative refractive index materials have opened more possibilities for the design of microwave devices. Generally included in a broader category of "metamaterials," these artificial composites owe their properties to subwavelength details of structure rather than to their chemical composition. Possible applications of this type of devices are based on the use of their unique features, such as backward wave propagation, ${ }^{1}$ negative refractive index, ${ }^{2}$ or evanescent wave regeneration. ${ }^{3}$ Even if to date the topic is still an emerging research line, one can cite, among others, promising potential applications such as imaging below the diffraction limit with the so-called "super-resolution,"4 cloaking, ${ }^{5,6}$ device miniaturization, ${ }^{7}$ or, very generally speaking, any unusual propagation effect not achievable with natural "materials."

In this context, a number of waveguide-based backward propagation devices have been proposed recently, starting from the original proposal in Ref. 8. In that paper, lefthanded or backward wave propagation was obtained for a structure composed of an array of split ring resonators $(\mathrm{SRRs})^{9}$ inside an evanescent waveguide. This composite structure possesses a left-handed transmission band slightly above the resonant frequency of the resonators, which is generated through the combination of a negative effective permittivity synthesized by the evanescent waveguide enclo-

\footnotetext{
${ }^{a)}$ Electronic mail: jorcarol@dcom.upv.es

${ }^{b)}$ Electronic mail: vboria@dcom.upv.es

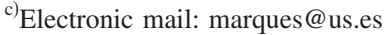

sure, and a negative effective permeability synthesized through the array of SRRs. After this pioneering paper, a number of studies followed using waveguide structures as the basis for their design. ${ }^{10-14}$ Yet, the common drawback of poor transmission performance, which could only be partially alleviated through the use of long tapering sections, (Ref. 15), was acknowledged. Two enhancements were theoretically introduced in, Ref. 16, which is a recent contribution aiming at improving the poor transmission levels previously reported. These can be cited as the use of a coaxial excitation to feed the waveguide structure and the use of inductive windows interleaved between the SRR array. This technology makes the transmission structure very compact in size with respect to other conventional waveguide configurations. The present paper analyzes both types of enhancements not only in terms of transmission, but also in terms of matching, interresonator coupling, location, and width of the pass band and loss contributions, thus, paving the way for their future use toward the applications. Furthermore, experimental results confirming the theoretical work have been obtained.

In this paper, Sec. II reviews the proposed structures, and the causes of performance improvement associated with the waveguide height reduction, with the use of the coaxial feeding probe and with the use of the inductive windows. Section III describes the design and manufacturing of a prototype and the experimental validation of the numerical predictions. Also, a measured result giving evidence of backward wave propagation is provided, together with a short discussion on 
the main features associated with the prototypes. Finally, Sec. IV summarizes the conclusions of this work.

\section{TOPOLOGIES FOR IMPROVING THE TRANSMISSION RESPONSE OF AN EVANESCENT WAVEGUIDE LOADED WITH AN SRR ARRAY}

All subsequent designs are based on a common SRR geometry. SRRs are all of the broadside-coupled type: one split ring on each side of the substrate and symmetrically oriented with respect to their axis. ${ }^{17}$ Broadside-coupled resonators do not present bianisotropy and have a reduced electrical size as compared to the particles proposed by Pendry in Ref. 9. Square-shaped devices were selected since performance was much better than for ring-shaped ones (better transmission and reflection characteristics), especially for the experimental results. This choice was justified because of the coupling improvement between the first resonator and the coaxial probe and also between neighboring resonators. Moreover, the fabrication process produced more accurate samples in the case of the square-shaped resonators. The lateral dimension of this "particle" is $l=3.2 \mathrm{~mm}$ and it has metallic track widths of $c=0.5 \mathrm{~mm}$. The ring gap or slit is $0.5 \mathrm{~mm}$ wide. These dimensions, patterned on a low permittivity substrate $\left(\varepsilon_{r}=2.2\right)$ with a thickness of $0.508 \mathrm{~mm}$, give an approximate resonant frequency of $f_{r}=8.5 \mathrm{GHz}$. The periodic array has a period of $p=4 \mathrm{~mm}$. For the simulations, if losses are included for the SRRs, finite conductivity of copper $\left(\sigma_{\mathrm{Cu}}=5.8 \times 10^{7} \mathrm{~S} / \mathrm{m}\right)$ and dielectric loss tangent for the substrate $(\tan d=0.0009)$ are taken.

\section{A. Influence of a reduced height waveguide}

Starting from the structure proposed in Ref. 12, the authors have first analyzed numerically the double influence of the use of reduced height waveguides instead of a full height (or standard) waveguide. Numerical simulations throughout this work use the commercial software simulator Ansoft HFSS. Standard waveguide dimensions for $X$-band operation correspond to those of a WR-90 $(22.86 \mathrm{~mm} \times 10.16 \mathrm{~mm})$, whereas the reduced height waveguide has a vertical dimension slightly bigger than the SRR dimension $(4.1 \mathrm{~mm})$. An analysis in terms of Sparameters shows that the use of a more-confined shielding structure around the resonators increases not only the amount of power being introduced inside the periodic structure, but also the interresonator coupling. This is directly observable in Fig. 1, since transmission and reflection levels are both improved. A transmitted backward wave band is observable close to the resonant frequency of the SRRs. The number of resonant frequencies in this transmitted band equals theoretically the number of resonators in the finite-length structure (4, in this case), however, the effect of material losses in the dielectric and conductors, combined with a high mismatch (represented by $S_{11}$ ), reduces the number of resonant peaks or dips. Moreover, reducing the height of the waveguide also increases interresonator couplings by widening the bandwidth of the backward wave band. The increased interaction between the resonators shifts upwards the individual resonant frequencies observable in the transmitted range. In both cases, feeding of the
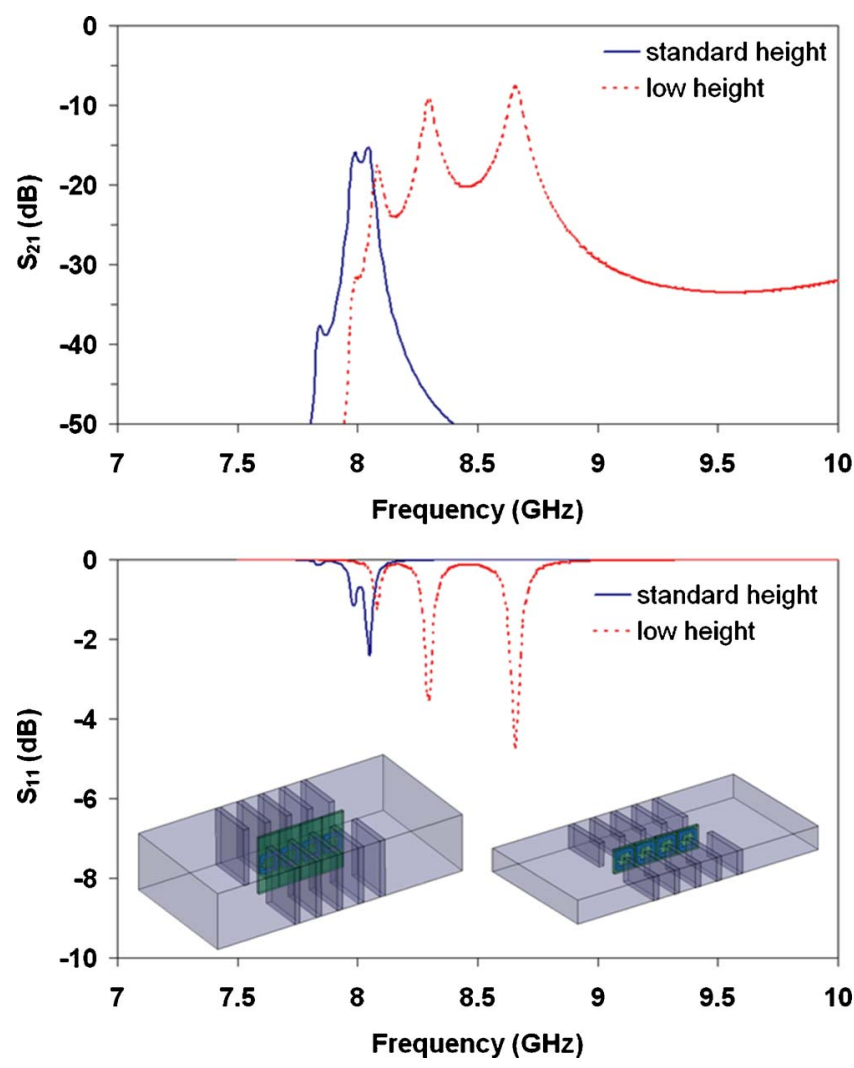

FIG. 1. (Color online) Reflection and transmission coefficients for a four split ring resonator backward wave structure in WR-90 including inductive windows. Insets show standard height and reduced height waveguide prototypes. These numerical results from full-wave simulations include metallic and dielectric losses in the resonators.

simulated structures is done directly through the definition of access ports at both ends, with their shapes corresponding to the waveguide transverse dimensions.

The excitation of the resonators inside the waveguide can be interpreted through the original fractional area concept introduced by Pendry for the SRRs in Ref. 9 (area of the SRR over area of the unit cell). It was shown in Ref. 18 that the coupling of the energy carried out inside the waveguide by the dominant $\mathrm{TE}_{10}$ mode to the resonator is based on this fractional area concept. Since the portion of the reduced height waveguide occupied by the SRR is bigger, much more magnetic-field lines will excite the resonators.

It is obvious that coupling between consecutive resonators also depends on the separation between them. There is a significant interaction among neighboring SRRs, but this only holds for small separation distances. Hence, it is the collective response of the array that influences the bandwidth of the device. Using a reduced height waveguide strengthens the interaction between consecutive resonators and the matching or excitation of the periodic structure, yielding an increased bandwidth.

\section{B. Coaxial probe excitation}

The use of a reduced height waveguide, as mentioned in the previous paragraph, precludes the use of a standard waveguide transition in order to feed the periodic structure, thus leading to the adoption of an optimized excitation 


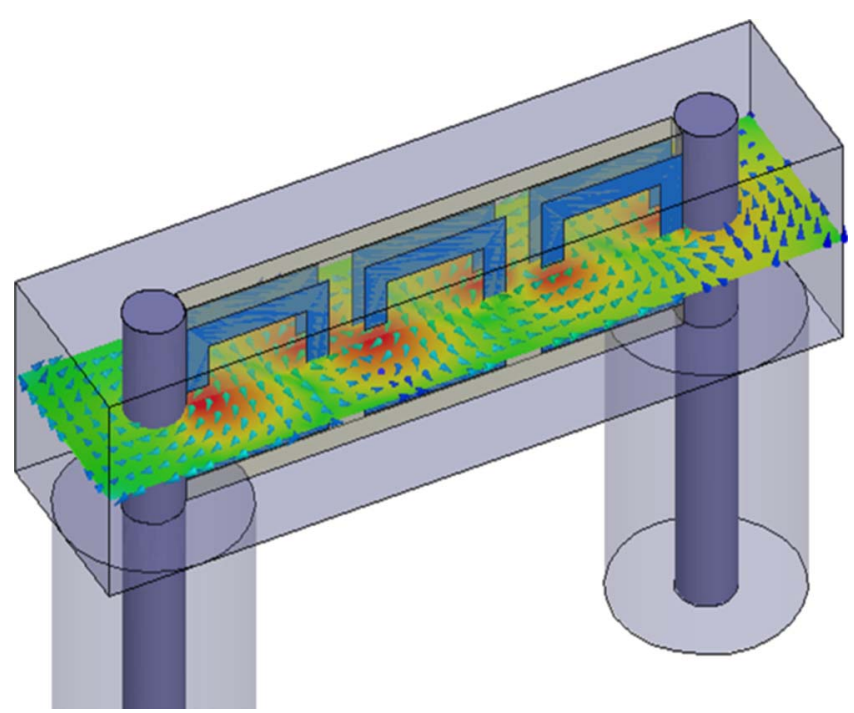

FIG. 2. (Color online) Magnetic field $\mathbf{H}$ generated from the short-circuited coaxial probe. Note the field orientation is aligned with the split ring resonator axis.

source for the periodic SRR array. Coaxial excitation of the device was then selected as an improved means of increasing a transmitted signal across an evanescent waveguide. At this stage, "evanescence" can be produced with periodic inductive windows, as shown in the insets of Fig. 1, or by directly using a waveguide with reduced transverse dimensions (width and height), which is the starting point of this subsection.

The description of the analyzed structure can be found in Ref. 16, as a waveguide approach drawn from the planar problem studied in Ref. 18. Let us recall that the unit cell size in all the periodic structures employed in the present paper is $4 \mathrm{~mm}$ (in the propagation direction), and the guided wavelength at $8.5 \mathrm{GHz}$ ( $X$ band) is $\lambda_{g}=55.5 \mathrm{~mm}$ in the WR-90. Roughly, the periodic structures have a relative unit cell size of $\lambda_{g} / 14$ as compared to the standard waveguide case.

Figure 2 illustrates the simulated magnetic field $\mathbf{H}$ generated in the longitudinal plane of a prototype structure with 3 SRRs. This prototype includes a short-circuited coaxial probe excitation feeding a SRR array in an evanescent waveguide. The very strong magnetic field generated is optimally oriented since it is parallel to the axis of the SRRs in the array plane. This provides enhanced coupling between the probe and the resonators, which causes the reduction of the reflection coefficient, and hence the improved matching of the structure.

In this configuration direct probe-to-probe coupling may affect the final transmission through the device. For these purposes, the structure without the metallic resonators, although including the dielectric substrate, has been simulated. Results (not shown) demonstrate that for a probe-to-probe distance of $7.2 \mathrm{~mm}$ (corresponding to a two-SRR device), the transmitted signal is not significant and is below $-50 \mathrm{~dB}$. The very evanescent waveguide enclosure precludes the direct transmission in the absence of the SRRs.

On top of the influence of the reduced height waveguide, the matching problem is clearly dependent on the distance
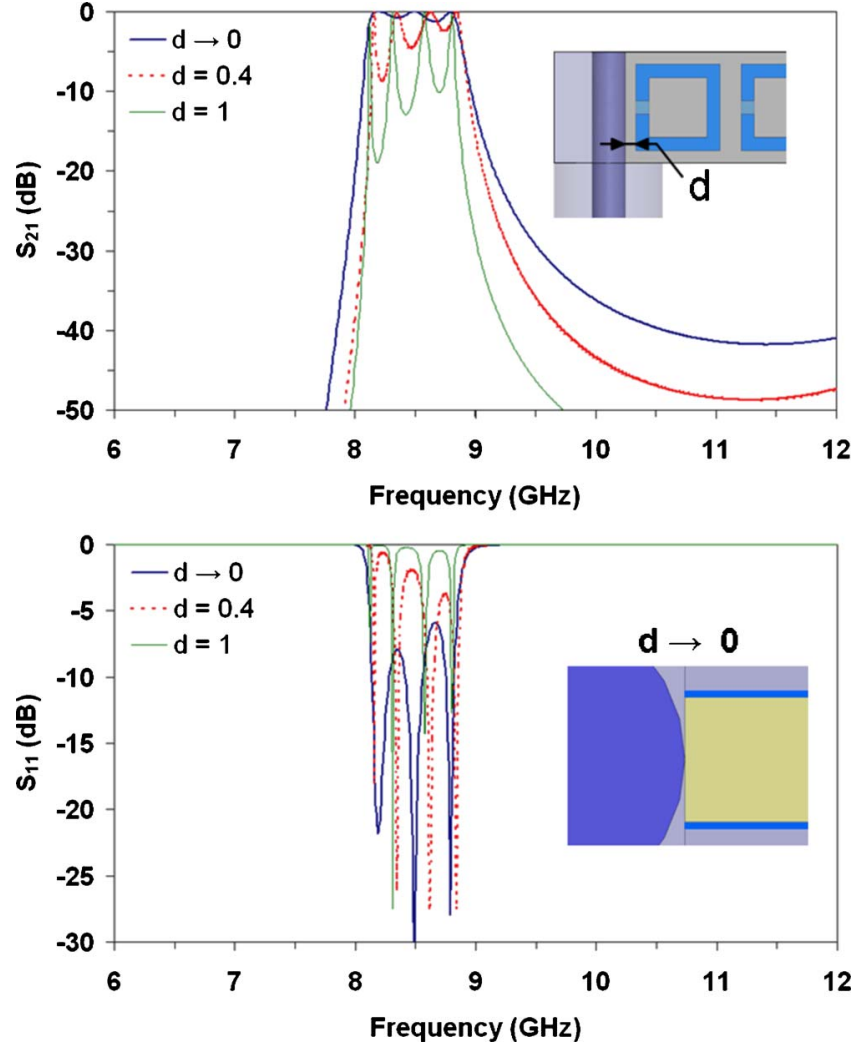

FIG. 3. (Color online) Numerical results for different values of the distance from the coaxial probes to the first and last resonators of the array (calculated transmission and reflection coefficients for a four SRR structure). Insets show lateral and top zoomed views of the design with indications on the distance from the probe to the first resonator.

from the probes to the first or last resonators of the array. This can be noticed in Fig. 3, which shows numerically computed $S$ parameters for a test structure with four SRRs. Material losses are not included in these simulations.

For three values of the distance from the first and last resonator to the coaxial probe $(d \rightarrow 0, d=0.4$, and $d=1 \mathrm{~mm})$, the plots show that enhanced matching, with a reduced reflection level, is achieved when the probe is closer to the resonators. This is apparent in the transmission curve where, for $d=1 \mathrm{~mm}$, very selective resonant peaks are defined in the transmission band. $S_{11}$ shows that only at these individual frequencies is the structure completely matched. Again, the number of these peaks corresponds to the number of resonators in the periodic structure. As the coaxial probe is closer to the dielectric substrate with the printed SRR tracks, and in the limit case both are "tangent" with $d \rightarrow 0 \mathrm{~mm}$ (Ohmic contact between the probe and the SRR rings, and hence, short circuits are avoided because of the circular shape of the coaxial probe, see the inset in Fig. 3), the selectivity of these resonant peaks is reduced with an increased overall transmission level. The reflection coefficient also shows an improved behavior with reduced level as compared to the previous cases. Coupling of the energy to the device is enhanced since the electromagnetic (EM) fields are evanescent inside the waveguide of reduced dimensions, and any small separation would imply a coupling loss. Only when they are very close to the probes, the first and last resonators strongly interact with them. The coaxial probes locally generate high EM 

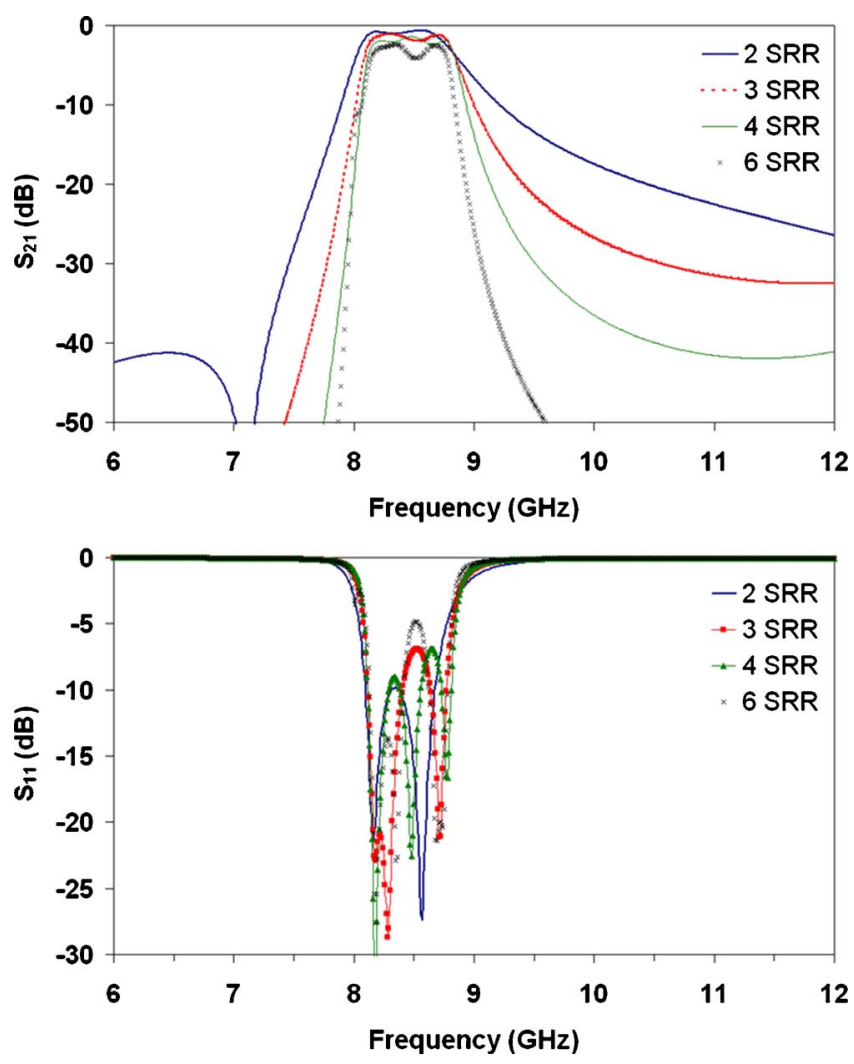

FIG. 4. (Color online) Transmission and reflection characteristics for different length periodic structures.

fields due to the short circuits with the waveguide enclosure. The strong current generated locally by the short circuit is, hence, contributing to the SRR excitation (which is essentially magnetic). It is also possible to observe in Fig. 3 that the out of band rejection level is reduced with a smoother fall of the transmission curve at high frequencies. Nevertheless, overall the transmitted bandwidth is in practice not dependent on this parameter.

The transmission and reflection characteristics for different length structures have been simulated in the case where the probes are quasi in contact ( $d \rightarrow 0$ case) with the first and last resonators of the array (see Fig. 4). Simulations do include losses in the conductors and the dielectric substrate. Transmission curves show a relatively high level band centered close to the resonance frequency of the SRRs with low losses. These losses slightly increase with the number of resonators but are smaller than $4 \mathrm{~dB}$ even for the case of the 6 SRRs structure. An estimate of the attenuation constant evaluated from the maximum transmitted level in the pass band for the different length structures gives an approximate value of $\alpha=1.06 \mathrm{~dB} / \mathrm{cm}$. This value clearly improves the performance of the results previously obtained by the authors in Ref. 12, which was around $\alpha=4 \mathrm{~dB} / \mathrm{cm}$, and is comparable to the results of Ref. 10 , claiming $a=0.8 \mathrm{~dB} / \mathrm{cm}$. It is important to note that for obtaining such low values external waveguide tuners were employed in this last work to match the evanescent structure. Also, an evanescent waveguide of a much larger cross section $(12 \mathrm{~mm} \times 12 \mathrm{~mm}$ against $4.1 \mathrm{~mm} \times 4.1 \mathrm{~mm}$ in this case) was used, hence, this implementation provides more compactness (operation frequencies

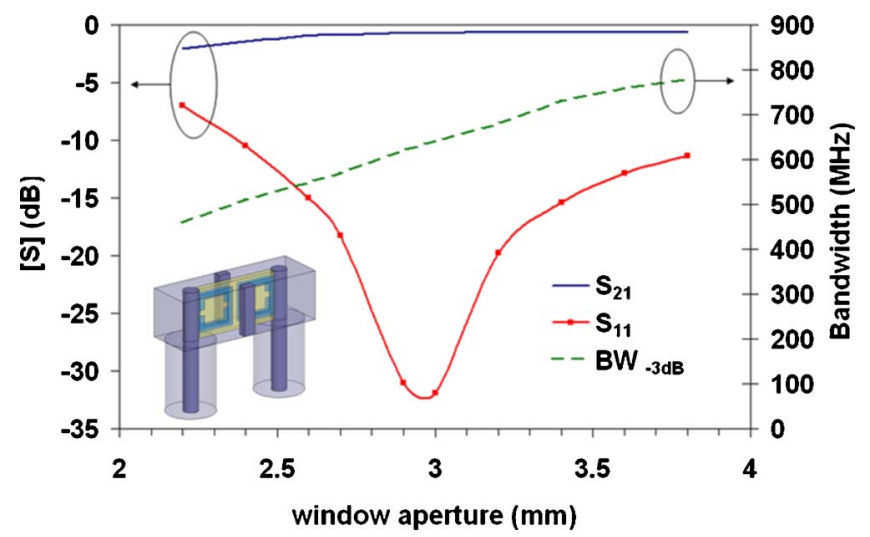

FIG. 5. (Color online) Transmission and reflection coefficients at the central frequency of the pass band and $-3 \mathrm{~dB}$ bandwidth as a function of the interleaved window aperture width for a two SRR device.

are in both cases close to $8 \mathrm{GHz}$ ). A lower evanescence of the waveguide in Ref. 10 lowers the attenuation constant, since interresonator coupling is based on proximity and evanescent fields. The bandwidth of the transmitted band remains constant with the length variations, whereas out of band levels decrease as the devices are longer, and hence, are more "selective." Fractional bandwidth can be estimated around $5.7 \%(0.5 \mathrm{GHz}$ bandwidth with $8.5 \mathrm{GHz}$ central frequency). These results are also in line with previously published results of devices based on split ring resonators (intrinsically narrow band structures), with, for example, 5\% bandwidth in Ref. 10. Main conclusion is, therefore, that the good matching provided by the coaxial probe feeding permits rather high transmission levels even for periodic structures sufficiently long to provide a good selectivity.

\section{Inductive windows}

The use of inductive windows or ridges in between the split ring resonators is a means of controlling the energy coupling between consecutive resonators, as it was stated in Sec. II A. Therefore, it is possible to include them in the prototype together with the coaxial feeding probe. This offers an extra degree of freedom in order to define, within a certain range of values, the reflection characteristics of the device as well as the bandwidth of the backward wave pass band. The window cannot have a larger aperture than the evanescent waveguide width. In this case, there would be no window and if the window were very tight (small aperture) the energy flow would be suppressed. For comparison purposes, the periodic array of SRRs of the previous section has been reused but including interleaved metallic windows inside the evanescent waveguide.

For a two SRR device, with one window interleaved between the resonators, Fig. 5 shows the numerical results in terms of $S$ parameters and bandwidth as a function of the window aperture. It is observable that there is an optimum window size that minimizes reflection losses giving an improved matching. This optimum value for the window aperture is slightly higher than $2.9 \mathrm{~mm}$. With respect to the transmission coefficient, $S_{21}$ has a flat characteristic with a high and quite constant level in a large range of window sizes. For 

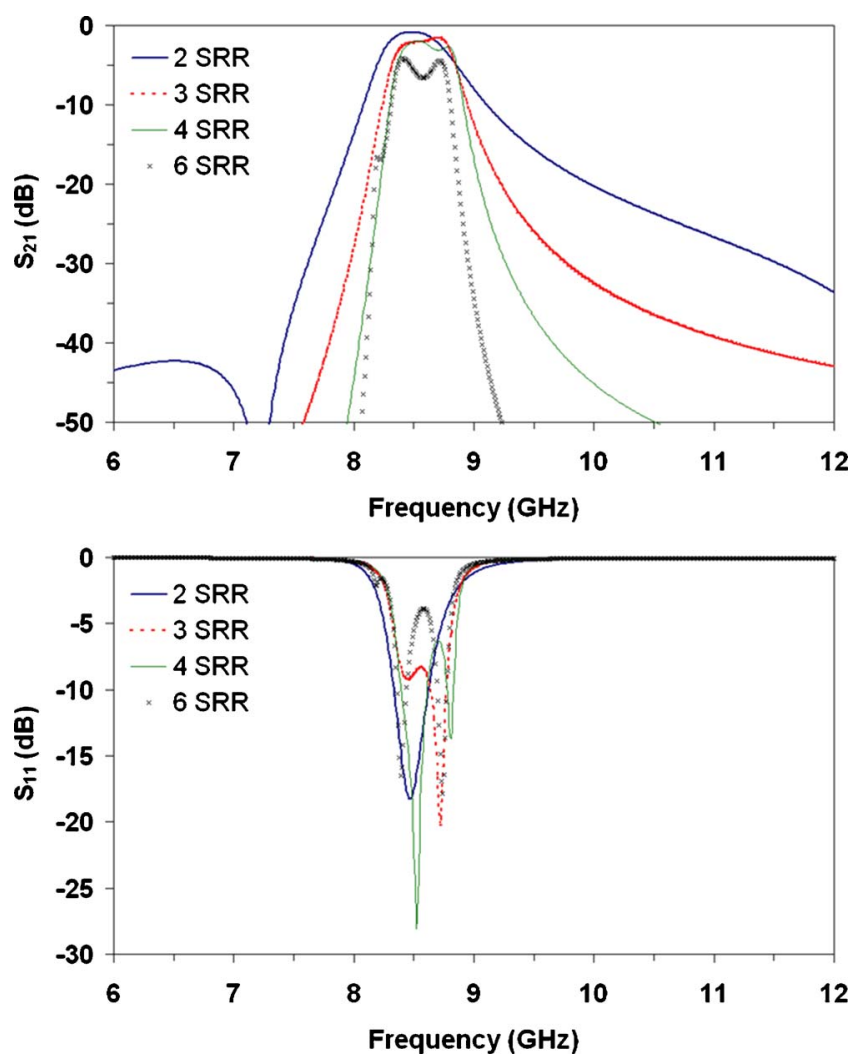

FIG. 6. (Color online) Transmission and reflection characteristics for different length periodic structures including interleaved inductive windows.

low values of the window width, the $S_{21}$ response is degraded with lower transmission. In terms of bandwidth, it is easily deduced that the bandwidth of the device increases together with the aperture width. This bandwidth variation is, hence, associated with the control of the interresonator couplings, just as in a classical design rule in filter theory.

Figure 6 shows the transmission and reflection characteristics for several structures with different number of resonators. The metallic ridges employed have a thickness of 1 $\mathrm{mm}$ and a lateral separation of $2.7 \mathrm{~mm}$; these are common values to all curves since the analysis is based on finite-size periodic structures. It is possible to observe that, by including the inductive windows, the two SRRs curve shows a very flat transmission band with a reduced in-band reflection loss. Generally speaking for the whole set of curves, bandwidth is reduced with respect to the curves in Fig. 4, and a certain control of the characteristics of the pass band can be achieved through the adoption of a specific window aperture value. The optimum values, maximizing transmission and minimizing reflection, for the window apertures depend on the number of resonators of the structure. Obtaining these optimum values would require employing, for example, filter design techniques, which is not the purpose of this work.

\section{EXPERIMENTAL VALIDATION AND DISCUSSION}

A prototype has been fabricated in order to validate the expected performance enhancement for the proposed features, see Fig. 7. The evanescent waveguide has transverse dimensions of $4.1 \mathrm{~mm} \times 4.1 \mathrm{~mm}$, and a segmented ap-

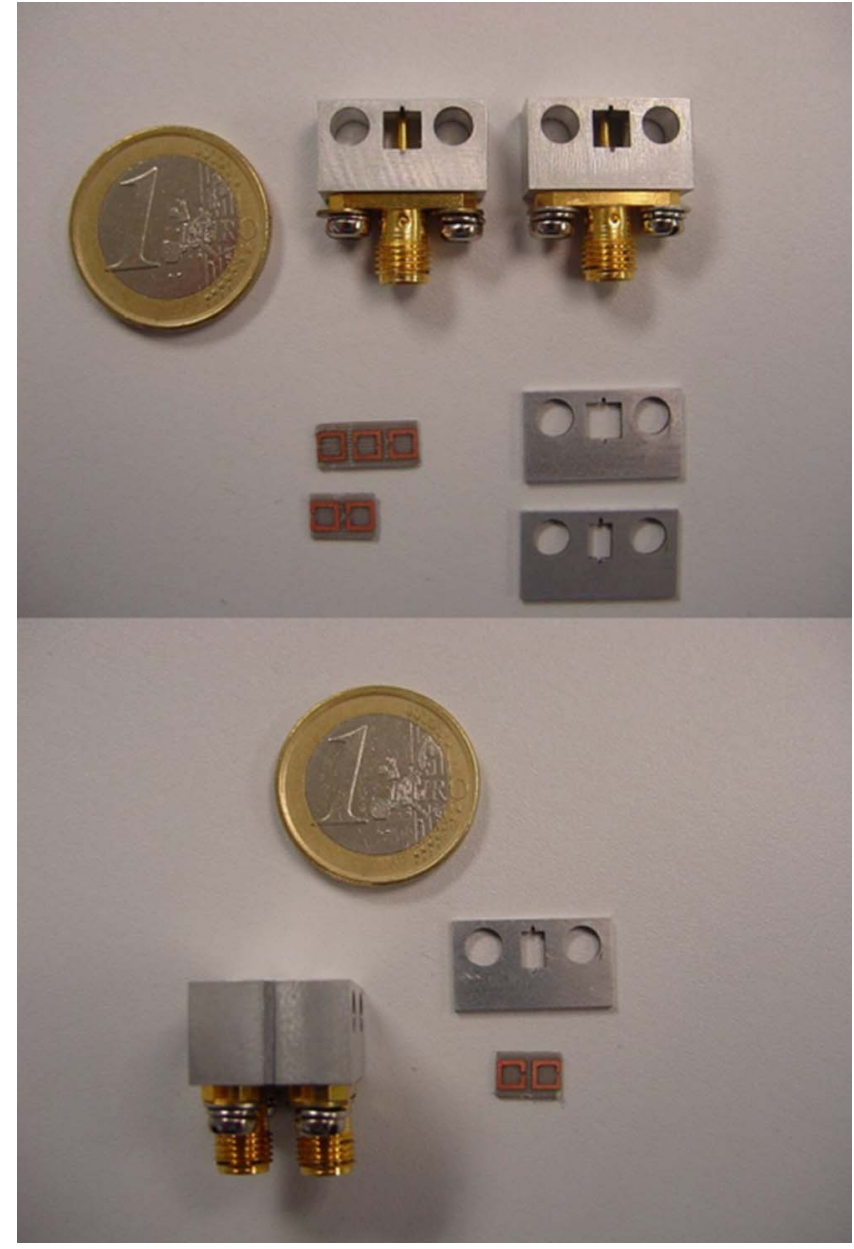

FIG. 7. (Color online) Segmented prototype composed of coaxial-toevanescent waveguide transitions, evanescent waveguide portions and square split ring resonator arrays (top); compact two SRR prototype with total length of $12.6 \mathrm{~mm}$ (bottom).

proach has been used in order to test different length devices, as in Ref. 12. Two segments of a full width evanescent waveguide and an interleaved window section are visible on the top picture of Fig. 7. Prototypes have been fabricated including either two or three resonators in the propagation direction. Also, two coaxial probe transitions have been implemented starting from standard coaxial SMA connectors inserted into the waveguide body terminations. These connectors have a long central conductor in order to assure the contact of the probe with the top of the evanescent waveguide producing a short. An interleaved window with an aperture width of $2.7 \mathrm{~mm}$ has been inserted between the resonators, a value close to the $S_{11}$ optimum point of the two SRR structure. This choice is justified in order to reuse the same window elements to measure a three split ring resonator line with moderately good transmission and reflection response. All the samples have been mechanically milled on a dielectric substrate of type Neltec NY9220 from Nelco, with the previously mentioned electrical and geometrical characteristics.

An HP 8510C Vector Network Analyzer has been used, with a standard coaxial through-reflect-line (TRL) calibration, to measure the $S$ parameters in the magnitude and phase of the prototypes. 

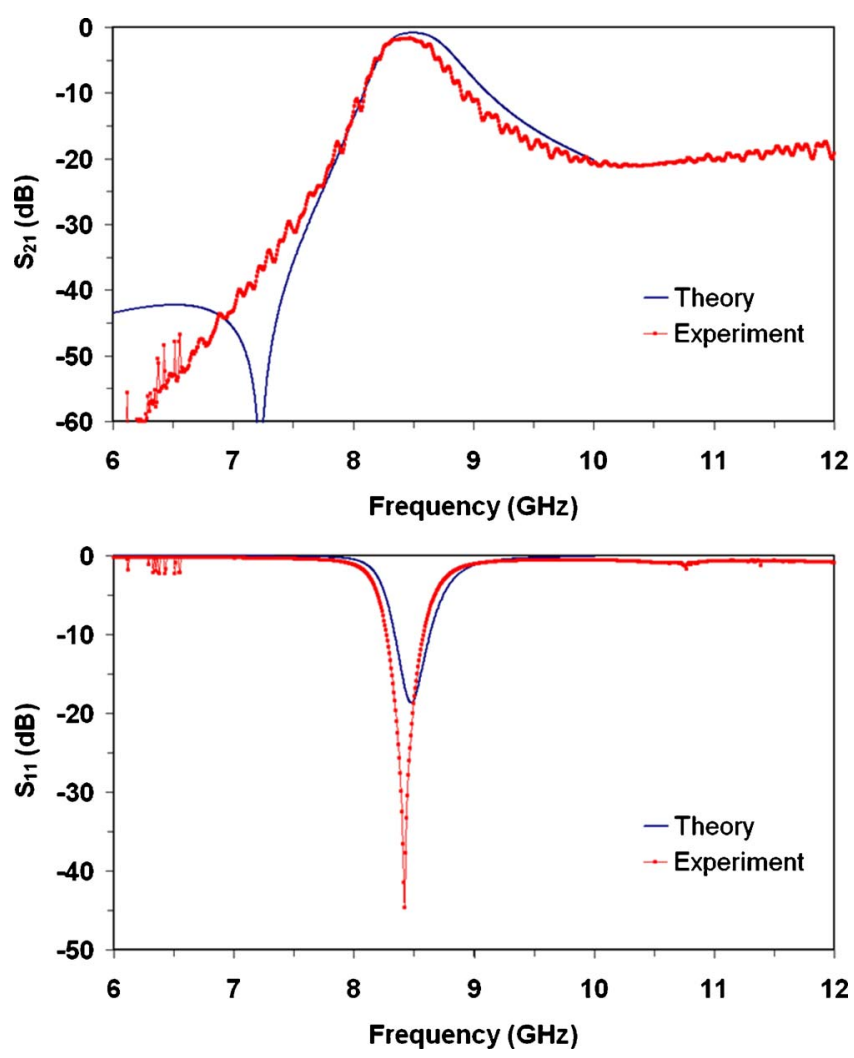

FIG. 8. (Color online) Reflection $S_{11}$ and transmission $S_{21}$ coefficients for a two SRR prototype, comparison between measured and numerical results.

Figure 8 shows a comparison of the numerical and measured results for a two SRRs device in terms of transmission and reflection coefficients. Both parameters show a good agreement between the expected and the measured characteristics, especially for the central frequency and the bandwidth of the pass band. The latter is limited due to the interleaved window effect and this fact results in the presence of only one resonance in the $S_{11}$ characteristic. Table I summarizes the numerical and experimental results obtained at the central frequency of the pass band. A very high transmission has been measured, combined with a moderate selectivity of the pass band and a very low reflection loss, thus alleviating the matching problems of previous implementations. Numerical results consider losses in the split ring resonators. ${ }^{19}$

In order to prove the left-handed or backward wave nature of this pass band, basically related to the split ring resonator behavior and the evanescent shielding, the measured

TABLE I. $S$ parameter comparison for two and three SRR devices.

\begin{tabular}{lcccc}
\hline \hline Structure & $S$-parameter & Type & $\begin{array}{c}\text { Value } \\
(\mathrm{dB})\end{array}$ & $\begin{array}{c}\text { Frequency } \\
(\mathrm{GHz})\end{array}$ \\
\hline 2 SRRs & $S_{21}$ & Measured & -1.71 & 8.48 \\
& & Simulated & -0.85 & 8.48 \\
& $S_{11}$ & Measured & -44.65 & 8.42 \\
& & Simulated & -18.64 & 8.49 \\
3 SRRs & $S_{21}$ & Measured & -3.90 & 8.70 \\
& & Simulated & -1.62 & 8.73 \\
& $S_{11}$ & Measured & -17.79 & 8.73 \\
& & Simulated & -19.70 & 8.73 \\
\hline \hline
\end{tabular}

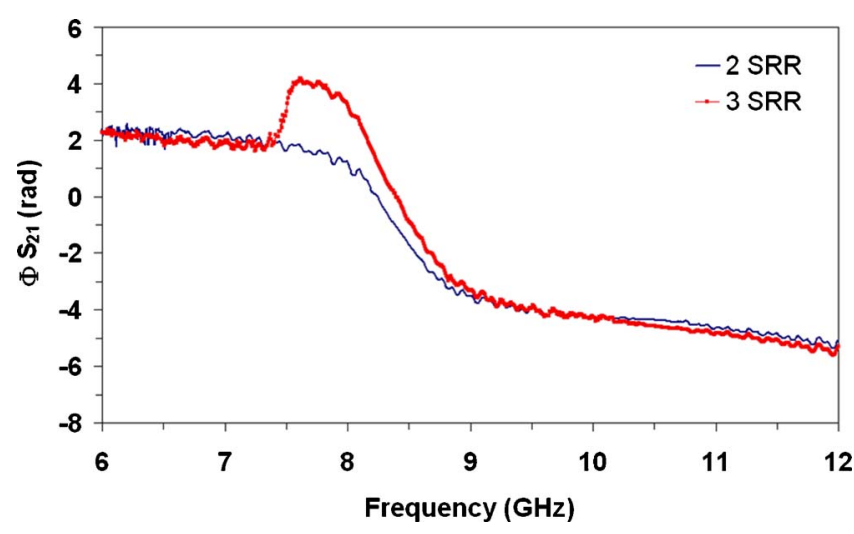

FIG. 9. (Color online) Unwrapped transmission $S_{21}$ phase measured for two and three SRR periodic structures including interleaved windows.

phase difference between two different length transmission lines $\left(l_{1}\right.$ and $l_{2}$, with $\left.l_{2}>l_{1}\right)$ is displayed in Fig. 9. It is possible to analyze the sign of the refractive index associated with a propagating structure with respect to the phase difference sign, analyzing it as taken from Ref. 20, which assumes the engineering convention $e^{j \omega t}$ for the variation of the electromagnetic wave

$$
\begin{aligned}
\Delta \phi & =\phi_{2}-\phi_{1}=-\beta l_{2}+\beta l_{1} \\
& =-\beta\left(l_{2}-l_{1}\right) \\
& =-\frac{\omega n(\omega)}{c}\left(l_{2}-l_{1}\right) .
\end{aligned}
$$

Then, if the phase difference is positive this would indicate the presence of a negative refractive index for the propagation of the EM wave. This is the case of the frequency range in Fig. 9, corresponding to the pass band of Fig. 8, i.e., 8.1-9 $\mathrm{GHz}$, which is in turn close to the resonance frequency of the resonators. Above this frequency range, for the rejected band where there is no transmission, both phase curves have equal values, hence producing a null phase difference with the consequence of prohibited propagation. Above approximately 10 $\mathrm{GHz}$, the phase difference would turn negative, then indicating a positive refractive index for a propagating band in an upper frequency range.

Figure 10 shows the measured characteristics $\left(S_{11}\right.$ and $S_{21}$ ) for a two SRR device including or not the interleaved window. In this comparison, the width of the evanescent waveguide is $4.1 \mathrm{~mm}$ and the window employed has an aperture of $2.7 \mathrm{~mm}$. While the variation in the transmission level in the pass band is small (around $1.4 \mathrm{~dB}$ in the middle of the pass band), the influence of the metallic window is great in terms of the reflection coefficient and important variations in the $S_{11}$ characteristic can be achieved. For instance, since there is a strong interaction between both resonators in the absence of the inductive window, two resonances are visible in the $S_{11}$ curve. Introducing the interleaved window limits this interaction and de-couples both resonators, giving just one resonance in the $S_{11}$ curve. In addition, pass band selectivity is increased with the use of the interleaved window with a sharper fall at both sides of the pass band. 

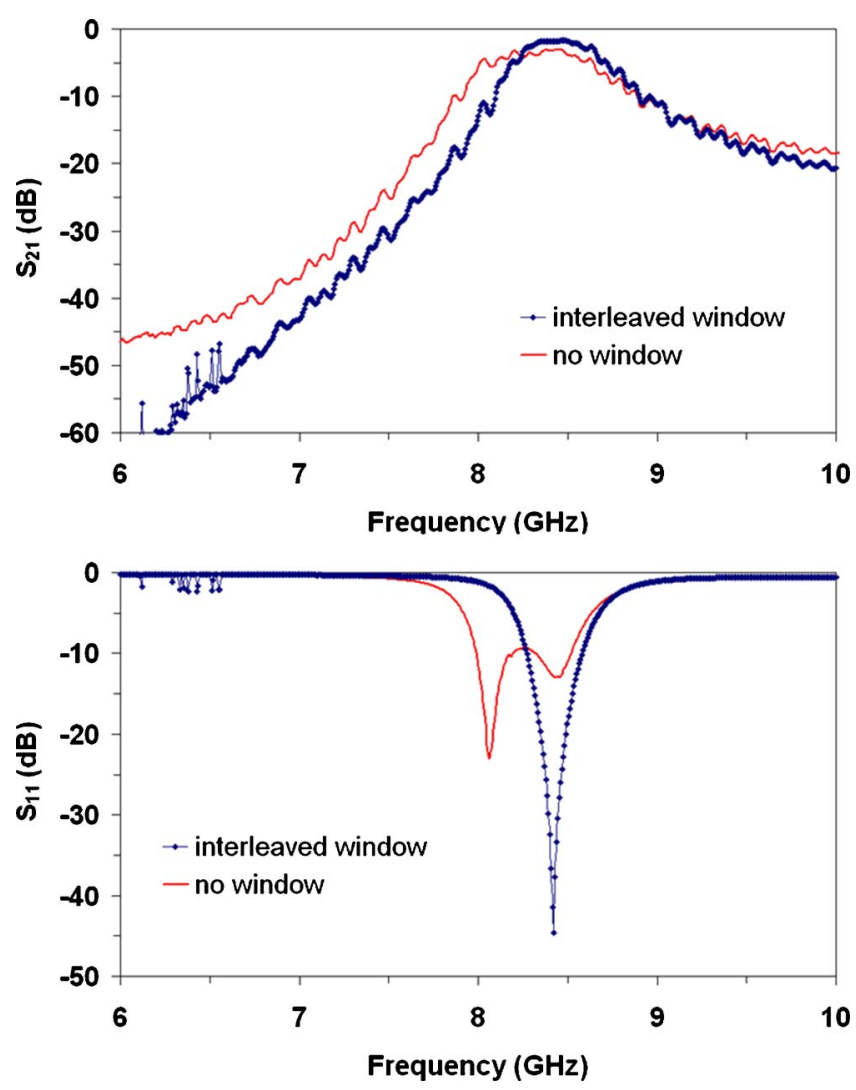

FIG. 10. (Color online) Measured transmission (top) and reflection (bottom) coefficients for a two SRR structure including or not an interleaved inductive window.

A three SRR structure has also been measured in terms of the $S$ parameters, with the results obtained shown in Fig. 11. The agreement between the predicted values of full-wave simulations and the measured data is reasonably good. The window size remains constant with respect to the two SRR prototype, and hence, it is not an optimum value in order to match the device. Nevertheless, for this periodic structure stronger selectivity has been obtained (with lower out-ofband transmission levels) and still a good transmission has been measured. Results are also included in Table I. It may be useful for the applications that a low number of resonators allow for a sharp fall of the pass-band edges. In contrast, and as predicted in the simulations, losses slightly increase with the number of resonators in the lines, but are still acceptable as compared to previous implementations of waveguidebased metamaterial lines. Discrepancy between theoretical and experimental values may be explained because of fabrication tolerances, since SRRs are quite sensible to the mechanical milling tolerances.

\section{CONCLUSIONS}

In this work, the very high losses associated with recently reported waveguide-based metamaterial transmission lines are partially overcome with the use of a direct coaxial excitation and the use of interleaved inductive windows inside a backward wave evanescent waveguide loaded with SRRs. These two features improve the transmission, reflection, and bandwidth characteristics of previously published
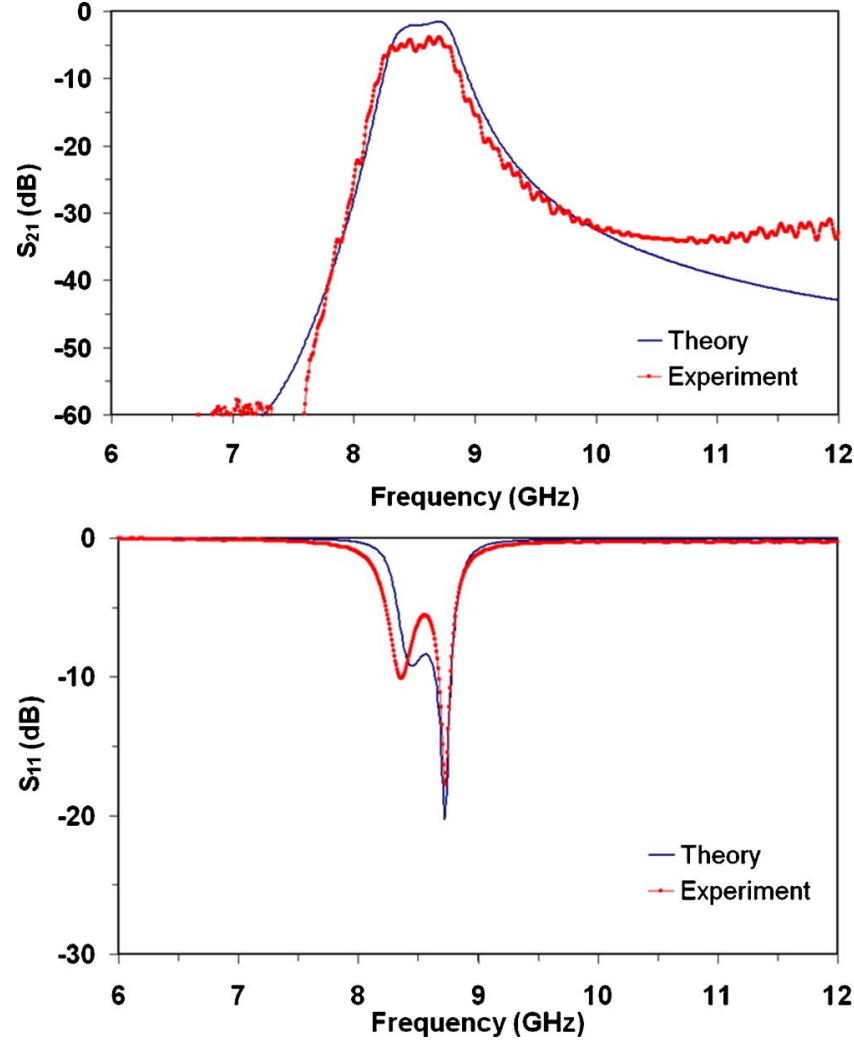

FIG. 11. (Color online) Reflection $S_{11}$ and transmission $S_{21}$ coefficients for a three SRR prototype, comparison between measured and numerical results.

prototypes and provide a means of controlling the basic characteristics of backward wave propagation in the periodic structures. Measured transmitted levels may allow the future use of this technology toward the applications. Also, this study opens the possibility to use standard synthesis techniques in order to move from periodic structures to regular filter designs, based on backward wave propagation. The main advantage of this type of potential technology is the very compact size of the analyzed prototypes.

\section{ACKNOWLEDGMENTS}

This work was supported by the Ministerio de Educación y Ciencia, Spanish Government, under a Ramón y Cajal Contract and under the Research Projects TEC-2004-04313C02-01 and TEC-2004-04249.C02-02 and by Generalitat Valenciana under projects ACOMP06/013 and GVA/2007/215. The authors are indebted to Bernat Bernardo for the fabrication of the SRR samples, and to Jose Vicente Castello for the preparation of the SMA connectors for the transitions.

${ }^{1}$ V. G. Veselago, Sov. Phys. Usp. 10, 509 (1968).

${ }^{2}$ R. A. Shelby, D. R. Smith, and S. Schultz, Science 292, 77 (2001).

${ }^{3}$ A. Grbic and G. V. Eleftheriades, IEEE Trans. Microw. Theory Tech. 51, 2297 (2003).

${ }^{4}$ J. B. Pendry, Phys. Rev. Lett. 85, 3966 (2000).

${ }^{5}$ A. Alu and N. Engheta, Phys. Rev. E 72, 016623 (2005).

${ }^{6}$ D. Schurig, J. J. Mock, B. J. Justice, S. A. Cummer, J. B. Pendry, A. F. Starr, and D. R. Smith, Science 314, 977 (2006).

${ }^{7}$ J. Garcia-Garcia, J. Bonache, I. Gil, F. Martin, Md. C. VelazquezAhumada, and J. Martel, IEEE Trans. Microw. Theory Tech. 54, 2628 (2006). 
${ }^{8}$ R. Marqués, J. Martel, F. Mesa, and F. Medina, Phys. Rev. Lett. 89, 183901 (2002).

${ }^{9}$ J. B. Pendry, A. J. Holden, D. J. Robbins, and W. J. Stewart, IEEE Trans. Microw. Theory Tech. 47, 2075 (1999).

${ }^{10}$ S. Hrabar, J. Bartolic, and Z. Sipus, IEEE Trans. Antennas Propag. 53, 110 (2005).

${ }^{11}$ I. A. Eshrah, A. Ahmed Kishk, A. B. Yakovlev, and A. W. Glisson, IEEE Trans. Microw. Theory Tech. 53, 3298 (2005).

${ }^{12}$ J. Carbonell, L. J. Roglá, V. E. Boria, and D. Lippens, IEEE Trans. Microw. Theory Tech. 54, 1527 (2006).

${ }^{13}$ C. A. Fernandes, R. Marqués, and M. Silveirinha Proc. European Microw. Association 2, 66 (2006)

${ }^{14}$ R. Marqués, J. Martel, F. Mesa, and F. Medina, Microwave Opt. Technol.
Lett. 35, 405 (2002).

${ }^{15}$ T. Decoopman, O. Vanbésien, and D. Lippens, IEEE Microw. Wirel. Compon. Lett. 14, 507 (2004).

${ }^{16}$ J. Carbonell, L. J. Roglá, V. E. Boria, and R. Marqués, Proceedings of the European Microwave Conference (IEEE, New York, 2006).

${ }^{17}$ R. Marqués, F. Medina, and R. Rafii-El-Idrissi, Phys. Rev. B 65, 144440 (2002).

${ }^{18}$ J. Carbonell, L. J. Roglá, and V. E. Boria Proc. European Microw. Assoc. 2, 52 (2006).

${ }^{19}$ M. J. Freire, R. Marqués, F. Medina, M. A. G. Laso, and F. Martin, Appl. Phys. Lett. 85, 4439 (2004).

${ }^{20}$ O. F. Siddiqui, M. Mojahedi, and G. V. Eleftheriades, IEEE Trans. Antennas Propag. 51, 2619 (2003). 